\title{
A reduced level of consciousness affects non-conscious processes
}

\author{
A. Fontan ${ }^{\mathrm{a}}$, L. Lindgren ${ }^{\mathrm{b}}$, T. Pedale ${ }^{\mathrm{a}}$, C. Brorsson ${ }^{\mathrm{c}}$, F. Bergström ${ }^{\mathrm{d}}$, J. Eriksson ${ }^{\mathrm{a}, *}$ \\ ${ }^{a}$ Department of Integrative medical biology, Umeå Center for Functional Brain Imaging, Umeå University, Sweden \\ ${ }^{\mathrm{b}}$ Department of Nursing, Umeå University, Umeå, Sweden \\ ${ }^{\mathrm{c}}$ Department of Anaesthesia and Intensive Care, Department of Surgery and Perioperative Sciences, Umeå University, Sweden \\ ${ }^{\mathrm{d}}$ Faculty of Psychology and Educational Sciences, University of Coimbra, Portugal
}

\section{A R T I C L E I N F O}

\section{Keywords:}

Consciousness

Unconscious

Sedation

fMRI

Brain activity

\begin{abstract}
A B S T R A C T
Being conscious is a profound aspect of human existence, and understanding its function and its inception is considered one of the truly grand scientific challenges. However, the nature of consciousness remains enigmatic, to a large part because "being conscious" can refer to both the content (phenomenology) and the level (arousal) of consciousness, and how these different aspects are related remains unclear. To empirically assess the relation between level and content of consciousness, we manipulated these two aspects by presenting stimuli consciously or non-consciously and by using Propofol sedation, while brain activity was measured using fMRI. We observed that sedation affected both conscious and non-conscious processes but at different hierarchical levels; while conscious processing was altered in higher-order regions (the intraparietal sulcus) and spared sensory areas, the opposite effect was observed for non-conscious processing. The observation that Propofol affected non-conscious processing calls for a reconsideration of what kind of information one can gain on "consciousness" from recording neural responses to sedation without considering both (content) conscious and (content) non-conscious processing.
\end{abstract}

\section{Introduction}

The concept of consciousness is multifaceted and can refer to at least two aspects: the content and the level/state of consciousness. The "content" relates to the core characteristic of consciousness, which is the subjective, phenomenal, "what-it-is-like" quality associated with experiencing something (Nagel, 1974). The level of consciousness commonly refers to arousal/wakefulness, and occurs on a continuum e.g., from comatose to fully awake (Laureys, 2005). These two aspects have mostly been investigated separately and there is much debate on how to conceptualize their relation (Bachmann, 2012; Bayne et al., 2016; Fazekas and Overgaard, 2016; Hohwy, 2009; Koch et al., 2016; Laureys, 2005; Overgaard et al., 2006).

On one hand, they can be considered as two aspects of the same underlying phenomenon (Aru et al., 2020; Bachmann and Hudetz, 2014; Mashour and Hudetz, 2017; Phillips et al., 2018; Suzuki and Larkum, 2020), which is supported by the observation that a certain level of arousal is required to enable conscious experiences. Indeed, we have a rich repertoire of conscious experiences when we are awake, and these experiences end during dreamless sleep or when we otherwise "lose" consciousness (Searle, 2000). In addition, brain research has demonstrated that the level of arousal affects integration of information across multiple brain regions (Casali et al., 2013), which has been proposed as fundamental for conscious experiences (Tononi et al.,
2016). Moreover, it has been suggested that neural mechanisms related to changes in the level of arousal overlap with the mechanisms generating conscious experiences (Aru et al., 2019). Yet, while general anesthesia, sleep, or coma, are commonly described as states that alter consciousness, the extent to which conscious experiences are lost/reduced when we are unresponsive is difficult to establish (Bayne et al., 2020; Fernández-Espejo and Owen, 2013).

On the other hand, the content and level/state of consciousness may be seen as separate phenomena (e.g., Bayne et al., 2016). A distinction between the two is apparent in every-day and clinical situations, which suggests instead that the level and the content of consciousness are not specifically interrelated. For example, vegetative-state patients can display sleep-wake cycles but remain unresponsive to external stimuli (Wislowska et al., 2017), and on rare occasions fully anesthetized patients can have conscious experiences (Errando et al., 2008). Moreover, we process information both consciously and non-consciously when we are awake (Axelrod et al., 2014; Kouider and Dehaene, 2007).

To better understand how level (hereafter referred to as "arousal") and content (hereafter referred to as "conscious perception") of consciousness are related, we set out to empirically assess their relation by manipulating both aspects while brain activity was measured using fMRI. Arousal was manipulated by administering two levels of the sedative Propofol. Importantly, participants were only mildly sedated and able to report whether they consciously perceived stimuli or not

\footnotetext{
* Corresponding author.

E-mail address: johan.eriksson@umu.se (J. Eriksson).
} 
and to perform tasks during both sedation levels. Within each sedation level, the content of consciousness was manipulated by presenting visuospatial stimuli both consciously and non-consciously. As a reference condition, we also included "absent" trials during both levels of sedation, where the subjective visual appearance during the trials was identical to non-conscious trials, but no target stimulus was presented (see Methods for details). This enabled us to isolate the blood-oxygenlevel-dependent (BOLD) signal change specifically related to conscious and non-conscious visuospatial neural processing, and to exclude the general, non-specific effects of sedation, including e.g., basal physiological processes. Two possible outcomes may be expected. If reduced arousal selectively reduces neural processing of consciously perceived stimuli, the neural processes related to conscious perception would be more or even uniquely affected by a change in arousal compared to nonconscious perception. Alternatively, neural processes would be affected by a change in arousal regardless of whether stimuli are consciously perceived or not.

\section{Materials and methods}

\subsection{Participants}

Forty healthy right-handed adults took part in the experiment. Participants were recruited from Umeå University campus through poster and internet advertisements. They had normal or corrected-to-normal vision, right-eye dominance, gave their written informed consent, and received financial compensation for participation (600 SEK). Eight participants were excluded from the analyses, either due to excessive head movement during fMRI scanning $(n=4)$ or for failing to follow task instructions $(n=4)$. Thus, the final sample in the analyses was 32 individuals (mean age \pm SD: $26.7 \pm 4.4$ years; 13 males). The relatively large number of excluded participants is likely related to the aim to implement the biggest possible reduction of arousal without having participants become too sedated to perform behavioral tasks reliably (see below). This study was approved by the regional ethics review board (dnr 2018-314-32 M).

\subsection{Procedure overview}

Each participant took part in three sessions spread out over a 1014 day period. On the first session, participants were trained on short versions of a visuospatial and a "metronome" task, to ensure that they understood the tasks and that the procedure with continuous flash suppression (CFS) worked as intended (see below for description of tasks and CFS). On the second session, personalized levels of Propofol sedation were identified (see section "Propofol sedation: individual adjustment" below for details).

On the third session fMRI data were collected (see Fig. 1A for MRI session overview). Propofol infusion started right before participants were placed in the scanner bore. Participants began the experiment with either low (LS) or moderate (MS) sedation. In the final sample, 18 participants started with LS and 14 with MS. A certified intensivecare nurse with specific responsibility for pharmacological administration and monitoring was present throughout the session, and complete resuscitation equipment was available at all times. The session started with structural imaging (T1, T2 FLAIR and T2 PROPELLER sequences) so that Propofol levels could stabilize before fMRI scanning. Then, two resting-state fMRI sequences were run, one at each sedation level, for the use of another study and will not be further reported here, and task fMRI followed.

During task-fMRI, participants performed two 7 min blocks of the visuospatial task under both sedation levels. Each block was followed by a 6 min stabilization period where sedation level was changed and during which participants performed the "metronome" task. This resulted in 4 blocks of visuospatial task and 3 stabilization periods. Finally, to verify that Propofol was not interfering with regional cerebral blood flow at the sedative concentrations (Veselis et al., 2005) and did not modify flow-metabolism coupling (Johnston et al., 2003), the MRI session included one pulsed arterial spin-labeling (ASL) sequences for each sedation level.

\subsection{Paradigm and stimuli}

\subsubsection{The visuospatial task}

The main task, performed during stable periods of Propofol infusion, consisted of noting the location of a gray disc presented in one of the display's quadrants (Fig. 1B). The simplicity of the stimuli reduces the issue of "partial awareness" (Kouider et al., 2010). Similarly, locating a stimulus in space is an uncomplicated task that seems to have a high probability to enable both conscious and non-conscious neural processing. There were three presentation conditions: a conscious, a non-conscious, and an "absent" condition. Conscious/non-conscious perception was manipulated with continuous flash suppression (Tsuchiya and Koch, 2005). The task was composed of 120 trials equally distributed in 2 blocks and divided into 3 presentation conditions: 40 conscious, 60 non-conscious, and 20 absent trials for each sedation level. Each trial was randomly chosen from one of the three conditions.

For CFS, a mirror stereoscope was used to isolate visual input from left and right side of the screen to participants' corresponding eyes. For non-conscious trials, the target stimulus (gray disc; size $=0.6^{\circ}$ ) was presented for $500 \mathrm{~ms}$ to the non-dominant (left) eye while colored squares of random composition ("Mondrians"; size $=4.2^{\circ} \times 4.2^{\circ}$ ) where flashed $(10 \mathrm{~Hz})$ to the dominant eye to suppress conscious experience of the disc. Mondrians were flashed for $500 \mathrm{~ms}$ longer than the disc's presentation, minimizing the risk of adaptation after-effects. To maximize stimulus intensity during non-conscious trials, contrast between the disc and the gray background was increased or decreased every 10 trials depending on how many times participant reported the disc as seen. That way, the proportion of actual non-consciously experienced disc presentations was $80 \%$. There were 17 possible contrast values. The difference between each contrast consisted of an increase or a decrease in RGB value of 2 (range $=174-206$; background $=210$ ). For conscious trials, the disc (RGB $=198$ ) was superimposed on Mondrians, presented to the dominant eye, and was thus consciously seen. For "Absent" trials, used as reference condition, Mondrians were presented to the dominant eye while an empty gray background $\left(4.2^{\circ} \times 4.2^{\circ}\right)$ was presented to the nondominant eye.

For conscious and non-conscious trials, the disc was presented in one of the four quadrants of the screen. The position was randomly selected from a pre-specified list where positions were counterbalanced within each condition. After the disc presentation, a probe was presented, pointing either to the same spatial location as the disc (match) or to another spatial location (non-match). Participants had to decide whether the probe was pointing to the disc's location (yes/no). For nonconscious and absent trials, participants were instructed to guess on the first alternative that came to mind. There was $50 \%$ chance that the probe pointed to the disc location. After the probe, participants estimated their conscious experience of the disc on a three-point perceptual awareness scale (PAS; Ramsøy and Overgaard, 2004), from 1: no visual experience to 3: clear visual experience of the disc. For probe and PAS, participants had to reply within a limit of $2.5 \mathrm{~s}$ after which the experiment automatically continued to the next trial. The inter-trial interval (ITI) was adjusted according to participants' response time in a way that two trials were always separated by $5 \mathrm{~s}$.

\subsubsection{The "metronome" task}

A visual metronome task was also performed and used as a behavioral measure of participants' arousal (Fig. 1C), and consisted of timing motor responses as synchronous as possible to a gray disc presented in one quadrant of the display. Participants were requested not to follow the beat by moving other body parts or using covert counting. The stimulus was the same disc as for the visuospatial task but presented on 
A)

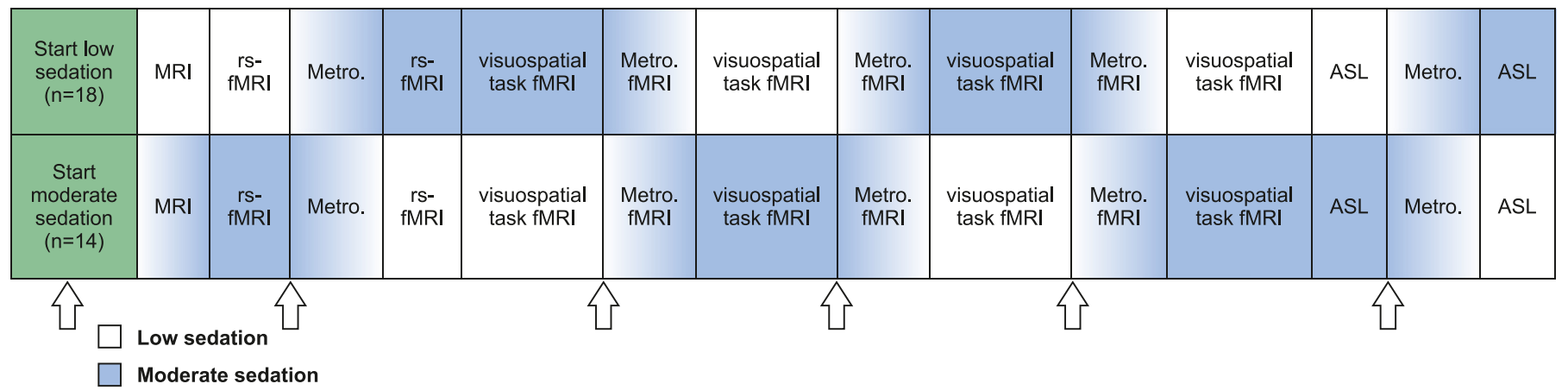

B)

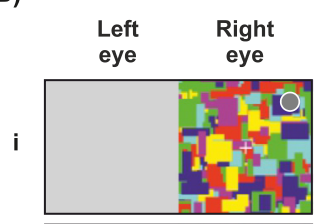

ii

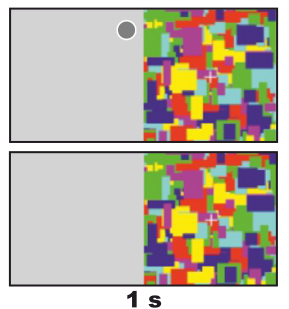

Visual experience
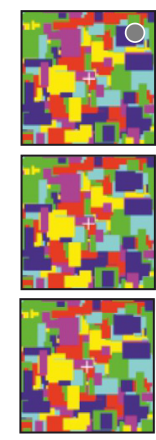

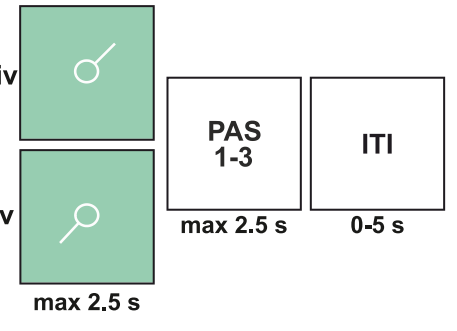

C) Sequence \#1 $\quad \ldots$ Sequence \#12

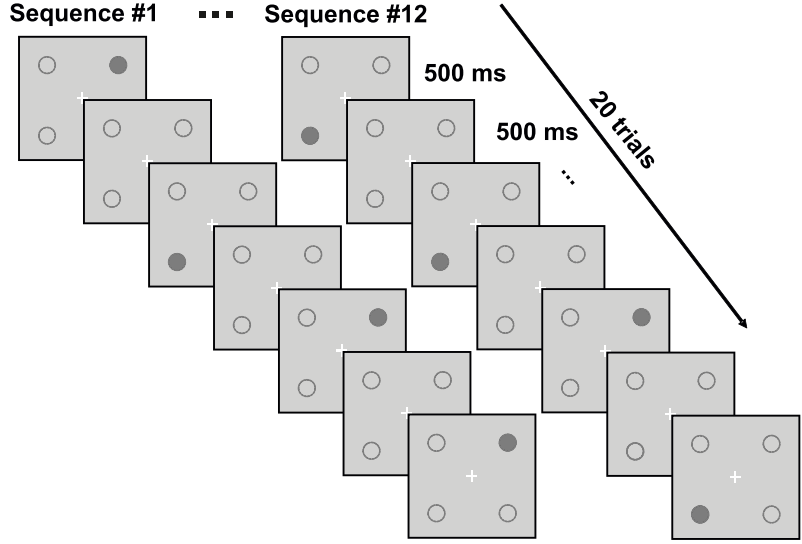

Fig. 1. Methods overview. (A) Sequence of events during fMRI scanning. Participants started with either low (upper row) or moderate (lower row) sedation. Each change in sedation is marked with an arrow at the bottom of the figure, leading to transitions from low (white) to moderate (blue) sedation, and vice versa, several times during the experiment. (B) The visuospatial task was composed of three presentation conditions. In each condition, Mondrians were displayed to the dominant (right) eye. (i) In the conscious condition, a gray disc was presented to the right eye, superimposed on the Mondrians. (ii) In the non-conscious condition, the disc was presented to the left eye, superimposed on a gray background and (iii) in the absent condition, only the gray background without the gray disc was presented to the left eye. The central column illustrates the visual experience of participants. After the $1 \mathrm{~s}$ Mondrian + disc presentation (note that the disc was presented for $500 \mathrm{~ms}$ during non-conscious trials, see Methods), a probe pointing to one of the four quadrants was presented for a maximum of $2.5 \mathrm{~s}$ or until participants decided whether it was pointing to the correct location of the disc (yes/no response). The probe could point (iv) to the correct location of the sample (match), or (v) to an incorrect location (non-match). Participants then estimated, within $2.5 \mathrm{~s}$, their conscious experience of the disc on a three-point perceptual awareness scale. Finally, trials were separated by an inter-trial interval (ITI). Note that for the purpose of illustration only, the gray dot is encircled in white. (C) The metronome task was performed after each change in sedation, to measure the effect of the change behaviorally and also to generate fMRI data on visuospatial stimulation independently from the visuospatial task. To this end, a gray disc was presented in a semi-blocked fashion in a specific quadrant throughout a sequence of 20 metronome "clicks". (For interpretation of the references to color in this figure legend, the reader is referred to the web version of this article).

the gray background with empty dotted circles reflecting the 4 possible positions of the stimulus apparition. Although the location of the pacesetting dot was irrelevant for the metronome task, this setup mimics the appearance of the stimuli in the visuospatial task and was designed in this way to be used as a ROI-defining sequence, in addition to gauge the effect of sedation behaviorally. One trial consisted of a $500 \mathrm{~ms}$ stimulus presentation followed by a $500 \mathrm{~ms}$ ITI. In total, participants completed 12 sequences of 20 trials ( 240 visual presentations) where the stimulus was presented with a $1 \mathrm{~Hz}$ tempo. The stimulus' position within each block was selected in a pseudo-random order in a way that a block mainly consisted ( $85 \%$ of the trials) of stimuli appearing in one quadrant. Finally, participants received feedbacks about their performance at the end of each sequence.

\subsection{Propofol sedation: individual adjustment}

The anesthetic agent used to manipulate arousal was Propofol (20 $\mathrm{mg} / \mathrm{ml}$ ), which activates GABAa receptors directly (O'Shea et al., 2000). Propofol is considered safe and fast acting (reaches its steady state $\sim 6$ min after infusion) (Trapani et al., 2000), which allowed us to change the level of arousal several times during the fMRI session.
Here, two sedation levels were used: a moderate level that was adjusted individually, and a low level $(0.1 \mathrm{mg} / \mathrm{kg} / \mathrm{h})$. The choice of having $0.1 \mathrm{mg} / \mathrm{kg} / \mathrm{h}$ rather than no sedative or saline injection, as a state of comparison was motivated by the fact that the sedative may affect blood flow or other non-neuronal parameters relevant to the fMRI signal (Qiu et al., 2017).

Individual adjustment of the moderate sedation level was evaluated during a pre-scanning session. Participants fasted from solids for at least $6 \mathrm{~h}$ and from liquids $4 \mathrm{~h}$ before sedation. Propofol was infused through an intravenous catheter placed into a forearm vein. Sedation was achieved using computer-controlled intravenous infusion of Propofol to obtain constant effect-site concentrations. Participants were initially injected with $2.0 \mathrm{mg} / \mathrm{kg} / \mathrm{h}$ of Propofol. The infusion rate was then increased in steps of $0.25 \mathrm{mg} / \mathrm{kg} / \mathrm{h}$, separated by a $6 \mathrm{~min}$ stabilization period, until participants were considered moderately sedated, operationalized as when they showed signs of having difficulties to keep their eyes open (i.e. increased frequency of blinks), but remained responsive in the sense that they could converse with the experimenters and perform the visual metronome task at all times. If participants presented difficulties to stay awake (i.e. dozed off, became unresponsive) or had to be prompted to continue performing the metronome task, the seda- 
tion level was considered too high and the previous dose was selected as the moderate sedation. Physiological parameters such as blood pressure, pulse oximetry, and breathing frequency were continuously monitored and were stable during Propofol infusion, and no side effects were observed. Anesthesia administration and monitoring were based on clinical judgment of the anesthesiologist and the intensive care nurse. In the final population $(n=32)$, the range of the moderate sedation was 2.25 to $4.0 \mathrm{mg} / \mathrm{kg} / \mathrm{h}$ (mean \pm SD: $2.8 \pm 0.5 \mathrm{mg} / \mathrm{kg} / \mathrm{h}$ ).

\subsection{MRI data collection}

MRI data were collected with a General Electric 3 Tesla Discovery MR750 scanner (32-channel receive-only head coil). High-resolution T1-weighted structural image was collected FSPGR with TE $=3.2 \mathrm{~ms}$, $\mathrm{TR}=8.2 \mathrm{~ms}$, TI $=450 \mathrm{~ms}$, and flip angle $=12^{\circ}$. Task-fMRI (1410 vol) was recorded using a $\mathrm{T} 2{ }^{*}$-weighted gradient echo pulse sequence, echo planar imaging, field of view $=25 \mathrm{~cm}$, matrix size $=96 \times 96$, slice thickness $=3.4 \mathrm{~mm}$. The volumes covered the whole cerebrum and most of the cerebellum containing 37 slices with $0.5 \mathrm{~mm}$ inter-slice gap and an ASSET acceleration factor of 2. The orientation was oblique axial, and slices were aligned with the anterior/posterior commissures, and scanned in interleaved order with $\mathrm{TE}=30 \mathrm{~ms}$, TR $=2 \mathrm{~s}$, flip angle $=80^{\circ}$.

Finally, ASL was collected using a field of view $=24 \mathrm{~cm}$, matrix size $=128 \times 128$, bandwidth of $62.50 \mathrm{kHz}$; slice thickness $=4 \mathrm{~mm}$. The acquisition orientation was axial aligned with the anterior/posterior commissures. The 40 slices with $2 \mathrm{~mm}$ inter-slice spacing were acquired from inferior to superior in an interleaved order to cover most of the cortex with a $\mathrm{TR}=4 \mathrm{~s}$.

\subsection{Data processing and statistical analyses}

In the visuospatial task, trials with response time (RT) $\langle 250 \mathrm{~ms}$ or $\rangle$ $2.5 \mathrm{~s}$ were excluded prior to statistical analyses (Ratcliff, 1993). Then, PAS responses between LS and MS during conscious or non-conscious trials were compared using multinomial logistic regression using a BeggGray approximation (Begg and Gray, 1984). Afterwards, only trials in absent and non-conscious conditions with PAS $=1$, and trials with $\mathrm{PAS}=3$ in the conscious condition were included in the following analyses.

For the accuracy analyses, a hit was defined as a position match between disc location and probe together with a "yes" response, while a "no" response was defined as a miss. False alarm (FA) was considered as a non-match between disc location and probe with a "yes" response, while a "no" response defined a correct rejection (CR). Accuracy was defined as the proportion of hits-FA for conscious and non-conscious trials.

Accuracy, under the two sedation levels, was compared using Wilcoxon's matched pairs test in conscious and $t$-tests in non-conscious conditions. The non-parametric test was chosen for the conscious condition because of the highly non-normal distribution, due to ceiling performance. RT differences between the two sedation levels were assessed using repeated-measure two-way ANOVA across the three visual presentation conditions. Specific differences for RT in MS and in LS between non-conscious and absent conditions were evaluated using Student's $t$ tests.

For the metronome task, the three first trials of each sequence and missed responses were discarded from analysis to include only trials where participants were synchronized to the stimulus. Visual-to-tap asynchrony was calculated as the absolute time difference between stimulus onset and participant's response. In other words, the smaller the difference, the better the performance. Because variability has been shown to be the most sensitive metric to capture performances' intra-individual changes with increase of cognitive fatigue (Wang et al., 2014), the variability in asynchrony was calculated for each sequence and each participant. Changes in variability due to Propofol sedation were estimated with the slope of a linear regression across the 12 sequences, and were used as a sedation-effect estimation. A positive slope (increased variability) with increased Propofol reflected a decrease in arousal and vice versa. To assess changes in the sedation effect over the three stabilization periods at the group level, the sign of the slopes related to participants who started the experiment with LS was switched, respectively for each stabilization period. Group level comparison was done using repeated-measure one-way ANOVA.

All post hoc tests with correction for multiple comparisons were conducted using the Holm-Bonferroni procedure and a $p$-value $<0.05$ was considered significant.

\section{7. fMRI analyses}

Image pre-processing, statistical fMRI, and ASL data analyses were conducted with SPM12 (Wellcome Department of Imaging Neuroscience, London, UK) running in Matlab 8.4 (Mathworks, Inc., Sherbon, MA, USA) using custom-made Matlab scripts. Functional images were (i) slice-time corrected, (ii) realigned to the first image of the time series to correct for head movement, (iii) unwarped to remove residual movement-related variance (Andersson et al., 2001), and (iv) co-registered to high-resolution structural data. Structural images were normalized to the MNI (Montreal Neurological Institute) template using DARTEL (Ashburner, 2007) and resulting parameters were used for functional images normalization, which were resampled to $2 \mathrm{~mm}$ isotropic voxel size. Finally, functional images were smoothed with an $8 \mathrm{~mm}$ FWHM Gaussian kernel.

Pre-processed data were analyzed using a two-stage summary statistics random effect model (Friston et al., 1995; Holmes and Friston, 1998). At the first stage, task-dependent changes in BOLD signal were modeled as zero-duration event regressors time-locked to (i) the Mondrians' onsets for the visuospatial task, including conscious, nonconscious and absent conditions for each Propofol level and each PAS rating, and to (ii) the stimulus' onsets for the visual metronome task, including the four stimulus positions. These regressors were convolved with the SPM12 canonical hemodynamic response function and entered into a general linear model (GLM). The models also included constant terms, 6 head movement parameters, nuisance regressors such as missed responses, and physiological noise ( 6 parameters) from white matter and cerebrospinal fluid, estimated using aCompCor method (Behzadi et al., 2007). Finally, a high-pass filter (cut-off $=128$ s) was applied to remove low-frequency drifts in the data. Contrast maps were computed on beta maps resulting from the estimated first-level GLMs, and individuals' maps were taken to second-level random-effects analyses (onesample $t$-tests) to account for inter-individual variability.

For the ASL data, the mean CBF value for gray matter for both sedation levels was calculated using histogram-based segmentation algorithm of the upper brain CBF values, based on the ASL sequences. Averaged difference images were converted to $\mathrm{mL} / 100 \mathrm{~g} / \mathrm{min}$ using a single-compartment model. CBF images were (i) co-registered to highresolution structural data, (ii) motion-corrected using a 6-parameters rigid body spatial transformation, and (iii) normalized to the MNI via DARTEL template image. CBF images for each participant were taken to second-level random-effects analyses (paired $t$-tests) to estimate CBF differences as a function of Propofol level.

Multiple comparisons correction of statistical maps at the second level was conducted on the whole brain using cluster-based extent thresholding of $p<0.05$ (FWE corrected) calculated based on the Gaussian random field method and following a cluster-defining threshold of $p<0.001$.

\section{Results}

\subsection{Sedation effect on behavior}

First, to ensure that participants' arousal was affected by Propofol sedation, we verified that the response variability relative to the 
A.

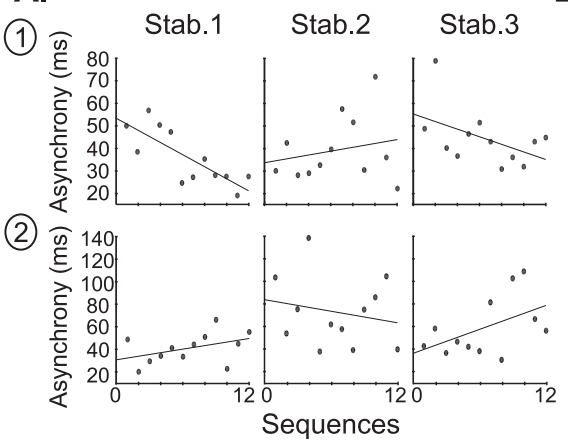

B.

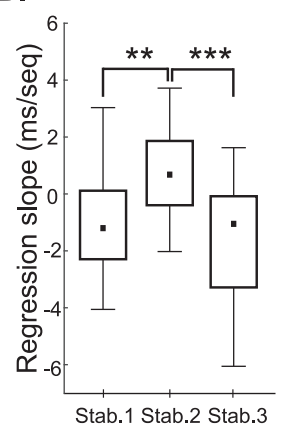

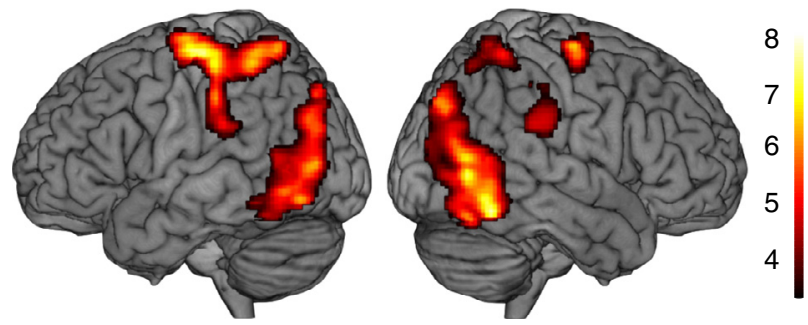

Fig. 3. BOLD signal change for Conscious $>$ Absent during low sedation. Bar values are $t$-values.

to $\mathrm{PAS}=2(\mathrm{OR}=0.93, p=0.75)$ for conscious trials. The odds of reporting stimuli as unseen (PAS $=1$ ) increased compared to PAS $=2$ during MS $(\mathrm{OR}=1.51, p=0.0001)$ but not compared to PAS $=3(\mathrm{OR}=0.88$, $p=0.52$ ) for non-conscious trials. The odds of reporting Absent trials as unseen (PAS $=1$ ) did not change between sedation levels (PAS $=1$ vs. $\mathrm{PAS}=2: \mathrm{OR}=0.98, p=0.90 ; \mathrm{PAS}=1$ vs. $\mathrm{PAS}=3: \mathrm{OR}=0.85, p=0.63)$.

To ensure no conscious visual experience in non-conscious trials and clear perception in conscious trials, only trials with PAS $=1$ in nonconscious (LS: $80.4 \pm 19.6 \%$; MS: $84.2 \pm 19.8 \%$ of trials) and in absent (LS: $86.8 \pm 17.0 \%$; MS: $85.9 \pm 17.5 \%$ of trials) conditions, and trials with PAS $=3$ in conscious condition (LS: $97.5 \pm 3.0 \%$; MS: $94.7 \pm 7.0 \%$ ), were included in the following analyses.

For conscious trials, participants had near perfect accuracy (hits false alarms; mean \pm SD: $L S=1.00 \pm 0.01 ; \mathrm{MS}=1.00 \pm 0.01$ ), with no difference between sedation levels (Wilcoxon match pairs test: $z=0.45$, $p=0.66$ ). For non-conscious trials, accuracy was at chance level (mean \pm SD: $\mathrm{LS}=0.02 \pm 0.12, t_{31}=0.89 ; p=0.38 ; \mathrm{MS}=0.01 \pm 0.12 ; t_{31}=$ $0.67 ; p=0.51$ ), again with no difference between sedation levels (paired samples $t$-test: $t_{31}=0.15, p=0.88$ ). As such, these stimuli were nonconscious according to both subjective (only trials with a PAS rating of 1 were included) and objective (chance-level performance on the forced-choice task) criteria. Participants' response time did not differ between non-conscious and absent trials for either sedation level (LS: $t_{31}=-1.41, p=0.17$; MS: $t_{31}=0.08, p=0.94$ ), but was generally slower during MS compared to LS (trial type-by-sedation ANOVA: main effect of sedation: $\mathrm{F}_{1,31}=15.18, p=0.00049$ ). There was also a main effect of trial type $\left(\mathrm{F}_{2,62}=44.78, p<0.0001\right)$, where conscious trials were faster than non-conscious $\left(t_{31}=-7.19, p=4.4 \times 10^{-8}\right)$ and absent trials $\left(t_{31}=-6.72, p=1.6 \times 10^{-7}\right)$. A significant trial type-by-sedation interaction $\left(\mathrm{F}_{2,62}=4.31, p=0.018\right)$ revealed that the effect of sedation was strongest on non-conscious trials $\left(t_{31}=-4.37, p=0.00013\right.$; conscious: $t_{31}=-2.97, p=0.006$; absent: $t_{31}=-2.55, p=0.016$ ), differing significantly from conscious $\left(t_{31}=3.95, p=0.00042\right)$ but not from absent trials $\left(t_{31}=-0.91, p=0.37\right)$.

\subsection{Neural response to stimulus presence}

We then investigated the neural response related to visuospatial processing at a low level of sedation, to verify task-related BOLD signal change in brain areas consistent with visuospatial processing (Wang et al., 2015) for both conscious and non-conscious stimuli, which could potentially be modulated by sedation. Whole-brain univariate analyses of fMRI data, contrasting the Conscious LS to the Absent LS condition, revealed significant BOLD signal change in the expected regions related with visuospatial processing, including superior parietal cortex, intraparietal sulcus, and occipitotemporal regions bilaterally (Fig. 3). Contrasting Non-conscious LS to Absent LS did not reveal any significant BOLD signal change at the whole-brain level. Such lack of significant signal change during non-conscious trials is not unexpected given previous research where continuous flash suppression (CFS) is used to suppress consciousness (e.g., Sterzer et al., 2014), or for non-conscious processing in general. Specifically, CFS produces powerful suppression and the multiple-comparisons corrections needed for whole-brain sta- 

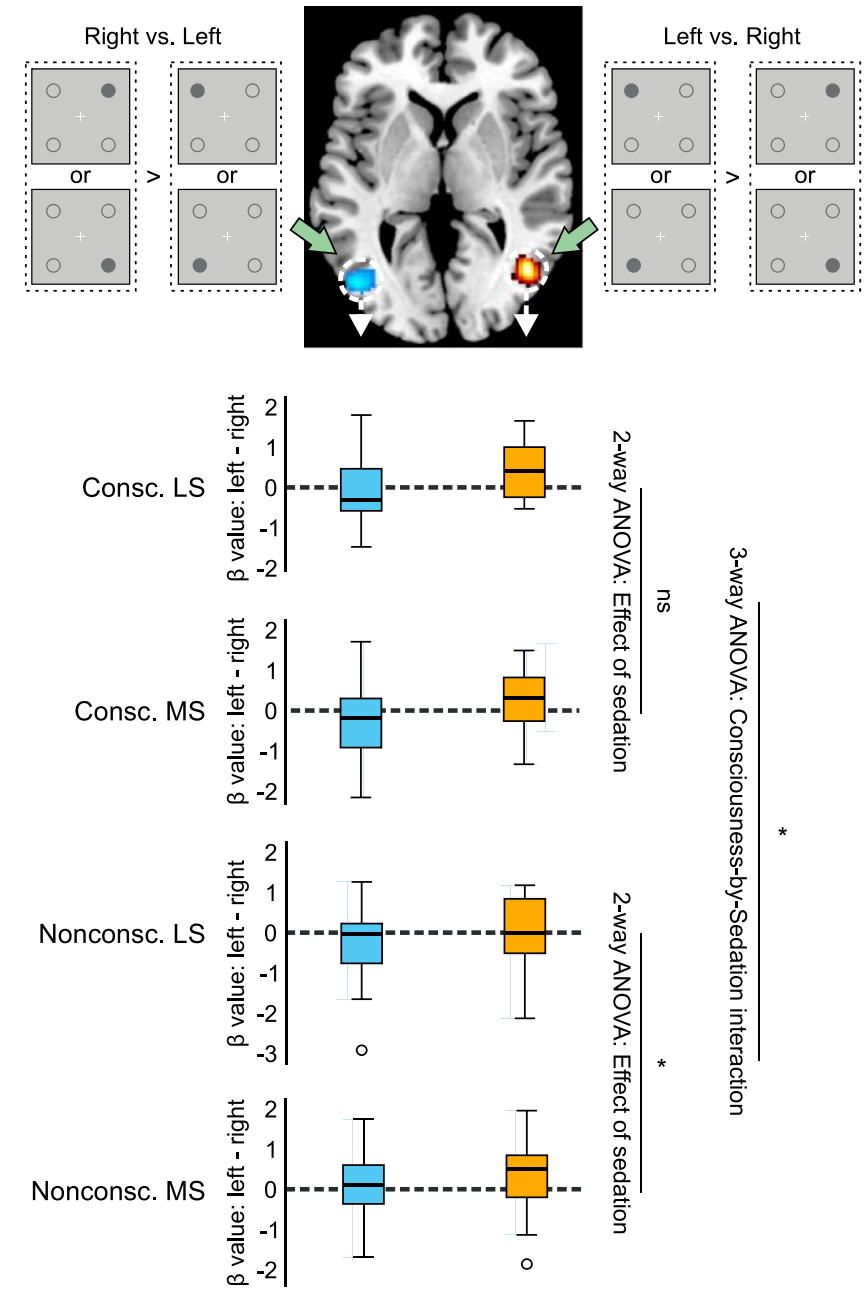

Left ROI Right ROI

Fig. 4. Regions of interest were defined based on the metronome task by comparing stimuli that appeared on the left vs. right side of the display (upper part of figure). Within each region, beta values from the visuospatial task related to right-sided stimuli were subtracted from beta values related to left-sided stimuli (y-axes in box plots, where the central line represents the median value). Thus, negative values are expected in the left ROI (where activity is driven more by stimuli appearing in the right visual field) and positive values are expected in the right ROI. Across these regions, beta value estimates were compared for conscious and non-conscious stimuli, when participants were under low (LS) or moderate (MS) sedation. The comparisons across sedation levels and across conscious/non-conscious conditions are from the ANOVAs described in the main text. Taken together, the results show that moderate sedation altered non-conscious and conscious neural processing differently. ${ }^{*}=p<0.05$. (For interpretation of the references to color in this figure, the reader is referred to the web version of this article.)

tistical inference makes such approach insensitive to detect a relatively subtle non-conscious signal change.

To optimize sensitivity and specificity when quantifying the signal change related to non-conscious perception, and later the effect of sedation on such signal, we limited further analyses to regions related to visuospatial processing. To generate regions of interest (ROIs) we used data from the metronome task, where the pace-setting dot appeared in one of the four quadrants of the screen. Capitalizing on the retinotopic organization of visual cortex, we compared left- > right-sided stimuli to define a region in the right hemisphere, and right- $>$ left-sided stimuli for a region in the left hemisphere $(p<0.05$, FWE corrected at the voxel level; Fig. 4). This resulted in a right-hemisphere region located in lateral occipitotemporal cortex (MNI coordinates: 46 -660) and a corresponding left-hemisphere region ( $-48-72$ 2). Importantly, the signal change in these two regions are expected to go in opposite directions for stimuli that appear on the left vs. right side of the screen, and the signal difference between regions would thereby constitute a more sensitive measure of stimulus processing compared with a consideration of signal change in each region by itself (cf. the "contralateral delay activity" commonly used in research on visual working memory; e.g., Luria et al., 2016).

Subtracting signal change related to right- from left-sided stimuli during the visuospatial task under low sedation (median beta value across voxels within each region), and comparing this difference across the two regions, revealed a significant hemispheric difference for both conscious $\left(t_{31}=3.46, p=0.002\right)$ and non-conscious $\left(t_{31}=2.59\right.$, $p=0.014$ ) stimuli (Fig. 4). Importantly, this latter finding confirms that the stimuli were processed also during suppression from consciousness.

\subsection{Sedation effect on neural responses}

To evaluate the effect of sedation on conscious neural processing, we submitted the BOLD signal change from the left/right ROIs to a Sedation-by-Hemisphere $(2 \times 2)$ repeated measures ANOVA. As expected, there was a main effect of Hemisphere $\left(\mathrm{F}_{1}, 31=17.52\right.$, $p=0.0002$ ). However, there was no effect from sedation (main effect of Sedation: $\mathrm{F}_{1,31}=1.62, p=0.21$; Sedation-by-Hemisphere interaction: $\mathrm{F}_{1,31}=0.074, p=0.79$ ).

The corresponding ANOVA on non-conscious neural processing revealed a main effect of Hemisphere $\left(\mathrm{F}_{1,31}=10.38, p=0.003\right)$, and, notably, also a main effect of Sedation $\left(\mathrm{F}_{1,31}=4.97, p=0.033\right)$. The Sedation-by-Hemisphere interaction was not significant $\left(F_{1,31}=0.12\right.$, $p=0.73$ ).

Taken together, the above results demonstrate that moderate sedation affected non-conscious and conscious neural processing differently (Fig. 4). To formally evaluate this difference across conscious/nonconscious conditions we performed a Consciousness-by-Sedation-byHemisphere $(2 \times 2 \times 2)$ repeated measures ANOVA. Indeed, the Consciousness-by-Sedation interaction was significant $\left(\mathrm{F}_{1,31}=5.80\right.$, $p=0.022$ ), confirming that sedation affects conscious and non-conscious neural processing differently in the ROIs. There was also a main effect of Hemisphere $\left(\mathrm{F}_{1,31}=25.02, p=0.00002\right)$ and a Consciousness-byHemisphere interaction $\left(\mathrm{F}_{1,31}=4.47, p=0.043\right)$. This latter interaction signifies a larger difference across hemispheres for conscious relative non-conscious stimuli. No other effects were significant. As can be seen in Fig. 4, there was an outlier in two of the non-conscious conditions. Exclusion of these participants did not change the outcome of the analyses.

Intuitively, it could be expected that an effect from increased sedation would primarily show as a Sedation-by-Hemisphere interaction, such that the lateralized response to left- and right-sided stimuli would decrease in both left and right hemispheres (i.e., both the left/"blue" and right/"orange" bars in Fig. 4 would shift towards zero, the left going up and the right going down), as a result of decreased signal strength and/or increased noise. Instead, the BOLD signal during non-conscious neural processing increased in both hemispheres (i.e., the main effect of Sedation). Given that the BOLD signal change was calculated as the difference between stimuli appearing on the left vs. right side of the screen, a common increase across hemispheres could be due to an increased response to left-sided stimuli, or a decreased response to rightsided stimuli, or both. As can be seen in Fig. 5, when re-plotting the data by subtracting MS from LS for each hemisphere and stimulus side separately, it is evident that moderate sedation changed the neural processing of left- and right-sided stimuli equally across hemispheres, such that the response to stimuli occurring on the left increased whereas the response to stimuli on the right decreased during MS compared with LS - i.e., a leftward bias. 


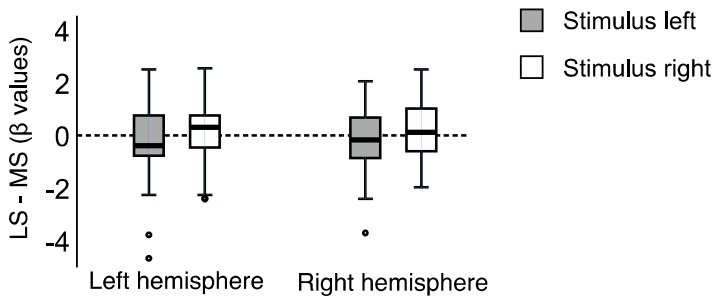

Fig. 5. Re-plotting of the effect of sedation on non-conscious neural processing from Fig. 4. By plotting the difference between low (LS) and moderate sedation (MS) for each hemisphere and stimulus side separately, it is evident that sedation alters the balance in response to left- vs. right-sided stimuli equally in both hemispheres. Note that the corresponding (Hemisphere-by-Stimulus Side) ANOVA provides the exact same statistics as displayed in Fig. 4, but here in terms of a main effect of stimulus side $\left(\mathrm{F}_{1,31}=4.97, p=0.033\right)$. This is because the input to the original ANOVA was the difference between Stimulus Left and Stimulus Right, while the current input data is the difference between LS and MS. Note also that an increased BOLD signal during MS will result in a reduced value from the subtraction LS - MS. That is, the boxplots move below zero when the response to left-sided stimuli increase during MS (gray boxes), and vice versa for a decreased response to right-sided stimuli (white boxes).

There is extensive evidence to suggest that the right hemisphere is dominant for spatial attention, with a concurrent left hemifield neglect in e.g. stroke patients (Corbetta and Shulman, 2011; Vuilleumier, 2013) and a positive leftward bias in healthy individuals (De Schotten et al., 2011; Jewell and Mccourt, 2000; Thut et al., 2006). We therefore hypothesized that there might be a change in attentional control with increased sedation, which could potentially explain the sedation-related increase in leftward bias during non-conscious neural processing. To investigate this, we first sought to find a possible neural correlate to "attentional control" of leftward bias during LS, by comparing left- vs. rightsided stimuli, and vice versa, for conscious stimuli (because there was no significant signal change in the whole-brain analysis for non-conscious stimuli, see above). Left $>$ Right trials during LS revealed a pattern of right-lateralized signal change in occipital cortex that overlapped with the right ROI from the metronome task, and in parietal cortex that overlapped with part of the activity pattern from Conscious $>$ Absent reported above (Fig. 6). Notably, the pattern of signal change included the right intraparietal sulcus (IPS), which is associated with attentional control and leftward bias (Siman-Tov et al., 2007; Vuilleumier, 2013). By contrast, there was no significant difference for Right $>$ Left trials. Comparing signal change in the right IPS (sphere with $3 \mathrm{~mm}$ radius at the local maxima within the right IPS, see Fig. 6) with the corresponding left IPS (defined based on the Conscious > Absent contrast), a Hemisphere-by-Stimulus Side ANOVA, with Stimulus Side flipped in one hemisphere, revealed a significant Hemisphere-by-Stimulus Side interaction $\left(F_{1}, 31=5.62, p=0.024\right)$. The significant interaction despite Stimulus Side being flipped in one hemisphere confirms a significant hemispheric difference, or "dominance" (cf. Shulman et al., 2010, for a similar approach). Moreover, BOLD signal in the right IPS was modulated by sedation, as revealed by a significant Sedation-by-Stimulus Side interaction $\left(F_{1,31}=6.97, p=0.013\right)$. Thus, the right IPS is a candidate region as a source of sedation-modified leftward bias.

The significant modulation of BOLD signal in the right IPS from moderate sedation is in itself an important addition to the findings presented above. However, since the signal change was occurring during conscious trials, an explanation to the leftward bias during non-conscious trials remains unfinished. Specifically, the right IPS would in addition need to be responsive to non-conscious stimuli. This was not the case, as $t$-tests during both LS and MS were non-significant (LS: paired $t$-test of Stimuli Left $>$ Right, $t_{31}=-0.61, p=0.55$; MS: $t_{31}=-0.46, p=0.65$ ). Per the same logic as presented above, a difference across hemispheres may be more sensitive to signal change related to lateralized stimulus presentations. Including right and left IPS in a Hemisphere-by-Stimulus Side ANOVA

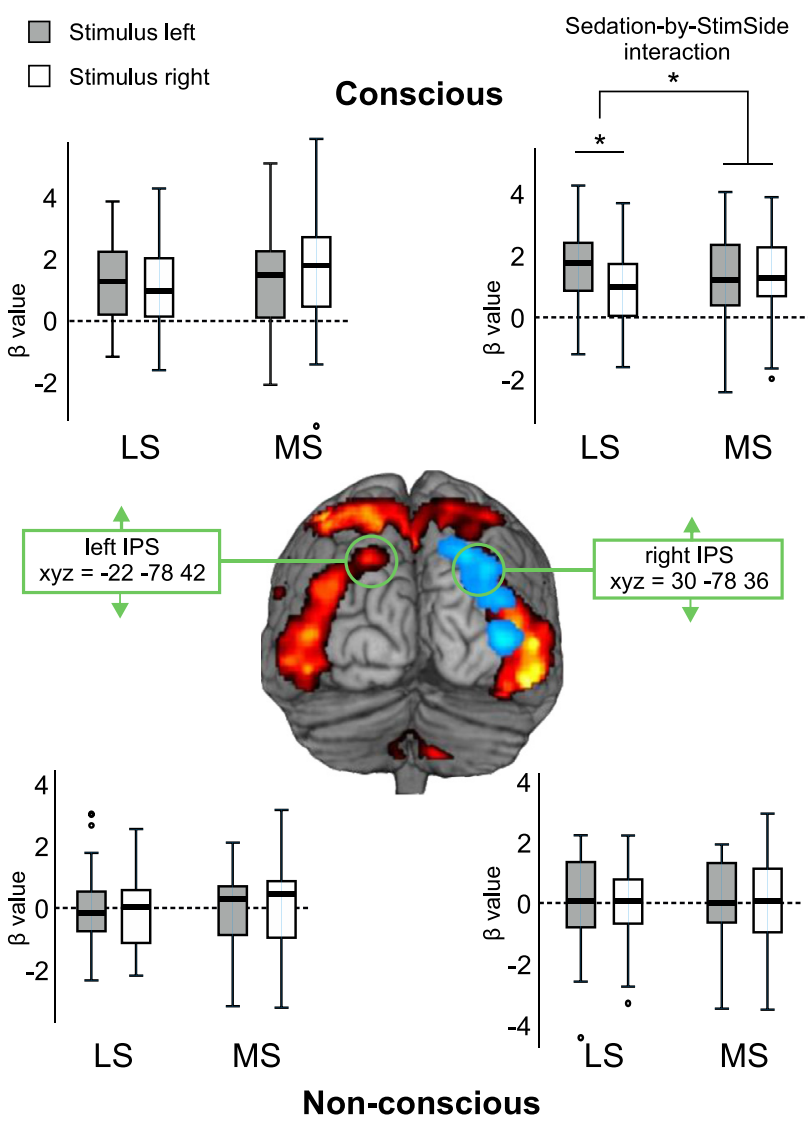

Fig. 6. Boxplots for BOLD signal change relative to the Absent conditions in left and right intraparietal sulcus (IPS) during low and moderate sedation (LS and MS, respectively), separately for stimuli appearing on the left (gray) and right (white) side of the screen. The brain rendering illustrates in hot colors the network of regions active during Conscious > Absent during LS (same as in Fig. 3), with Stimuli Left $>$ Stimuli Right during Conscious LS trials overlaid in cool colors. The reverse contrast of Stimuli Right $>$ Stimuli Left did not reveal any significant results. Neither IPS region was responsive to non-conscious stimuli (bottom row).

did not provide any significant results for non-conscious trials (LS: main effect of Hemisphere, $\mathrm{F}_{1,31}=0.18, p=0.68$; main effect of Stimulus Side, $\mathrm{F}_{1,31}=0.66, p=0.42$; Hemisphere-by-Stimulus Side, $\mathrm{F}_{1,31}=0.07$, $p=0.79$; MS: main effect of Hemisphere, $\mathrm{F}_{1,31}=0.33, p=0.57$; main effect of Stimulus Side, $\mathrm{F}_{1,31}=0.04, p=0.84$; Hemisphere-by-Stimulus Side, $\left.\mathrm{F}_{1,31}=1.90, p=0.18\right)$. We thus conclude that sedation modulates BOLD signal in sensory regions (the ROIs, see Fig. 4) during nonconscious trials by biasing the lateralized stimulus response towards leftsided stimuli, and that the origin of this bias remains unclear.

To explore if sedation might alter brain activity related to conscious neural processing outside the current ROIs, we also performed a wholebrain analysis of [Conscious LS - Absent LS] $>$ [Conscious MS - Absent MS] $(p<0.05$ FWE, cluster-defining threshold $=0.001)$. There was no significant signal change. However, when comparing LS to MS across all conditions (Conscious, Non-conscious, and Absent), there was a significant reduction of BOLD signal in the inferior occipitotemporal cortex during moderate sedation ( $x y z=-44-48-20$, peak $t=4.79, k=275$ ).

Finally, non-specific changes in cerebral blood flow (CBF) were quantified with Arterial Spin Labeling (see Methods). There was a widespread reduction of CBF in frontal, parietal, temporal, and cerebellar regions $(p<0.05$ FWE corrected, cluster-defining threshold $=0.001$; Fig. 7). Reduced CBF was also evident in the ROIs used in the analyses of BOLD signal change above (CBF averaged across ROIs: $t_{31}=3.90$, 


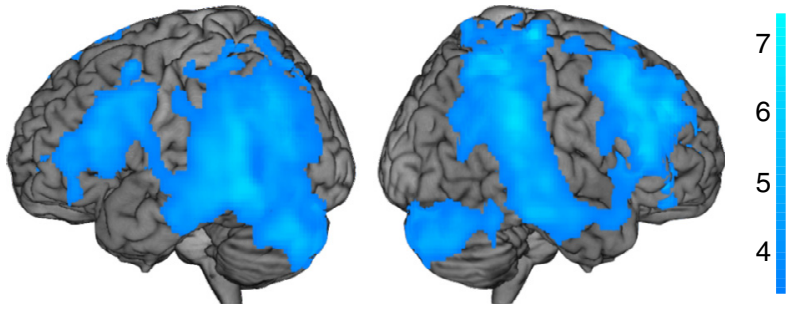

Fig. 7. Reduced cerebral blood flow from moderate sedation, as measured with arterial spin labeling during rest. Bar values are $t$-values.

$p=0.00049$ ). Importantly, sedation-induced changes in CBF did not correlate with changes in non-conscious neural processing $\left(r_{30}=0.26\right.$, $p=0.15$ ), and the main effect of sedation on non-conscious neural processing remained significant when adding $\mathrm{CBF}$ change as a covariate to the previous analysis of BOLD signal change (i.e., an ANCOVA: $\mathrm{F}_{1}$, $30=7.29, p=0.011$ ). This confirms that the approach to subtract the signal change in the Absent condition successfully controlled for nonspecific changes of sedation in the preceding analyses.

\section{Discussion}

The finding that a reduced level of arousal suppresses brain activity regardless of condition (Conscious, Non-conscious, and Absent), and the widespread reduction of CBF during resting state (ASL), is consistent with a bulk of previous research that has compared brain activity during sedated and unsedated conditions (e.g., Macdonald et al., 2015). Our current contribution to the field is to clarify how reduced arousal alters brain activity specifically related to conscious and non-conscious stimulus processing.

The most striking finding in our opinion is that reduced arousal affected non-conscious neural processes, and that this effect differed from the effects of Propofol on conscious neural processes. Specifically, for non-conscious stimuli Propofol sedation changed the balance of the lateralized response from left- and right-sided stimuli, thus increasing a leftward bias. There is substantial evidence for an attentional bias towards the left based on data from both healthy participants (De Schotten et al., 2011; Jewell and Mccourt, 2000; Siman-Tov et al., 2007) and neurological patients (Corbetta and Shulman, 2011; Vuilleumier, 2013). This body of research commonly point to parietal regions as a key source of such bias. Our attempt to explain the increased leftward bias as resulting from higher-order control of spatial attention, by investigating the BOLD signal change in the right IPS, was unsuccessful. It is noteworthy, however, that Propofol sedation reduced CBF in parietal and frontal regions, as evidenced by the ASL data. We speculate that this non-specific decrease in brain regions that overlap with several attention networks (Miller and Buschman, 2013; Petersen and Posner, 2012) may alter the cognitive context in which stimuli are processed non-consciously, which may have led to the observed increase in leftward bias. Alternatively, the bias may result from "preattentive" processing and would by such account reflect processing within sensory regions. However, the fact that the bias was symmetric across hemispheres/ROIs, combined with the retinotopic organization of ventral visual regions which would predict a lateralized effect, makes such a scenario less likely.

While the IPS was unresponsive to non-conscious stimuli, we found that IPS activity was modulated by moderate sedation during conscious trials. Interestingly, the differential response to stimuli appearing on the left vs. right side of the screen basically disappeared during moderate sedation, making the IPS equally responsive to left- and right-sided stimuli (Fig. 6). The altered IPS activity is consistent with a report by Henke and Schwarzbauer (2001), where they used subanesthetic levels of isoflourane combined with a visual search task. As in their report, sedation modified IPS activity and reaction time increased, while accuracy was unaffected. Different subsections of the IPS has been suggested to implement different aspects of attention (Vuilleumier, 2013), and the specific contribution of IPS to the current task is unclear.

The finding of altered neural processing of non-conscious stimuli from Propofol sedation has several implications for the concept of consciousness and for clinical situations. Firstly, it could be argued that the conception of "levels of consciousness" when referring to arousal is a misnomer. To denote a reduced level of arousal as an altered level (or state) of consciousness is potentially misleading, because such terminology suggests a specificity that apparently is non-existent. In light of our current findings, the correct term would be an "altered level of consciousness and unconsciousness". It may be more appropriate to simply use "arousal", "alertness", or similar terminology, because a change in arousal does not only affect conscious neural processing. Our findings are consistent with theories of consciousness that explicitly separate levels and content (e.g., Northoff and Huang, 2017), but are problematic for hypotheses that suggest integrating these two dimensions as subtending consciousness (e.g., Aru et al., 2019; Bachmann and Hudetz, 2014).

Secondly, our findings have implications for the development of "consciousness markers" in people with low levels of arousal and/or altered "states of (un)consciousness". One great challenge in consciousness research, which has substantial ethical implications, is to know whether patients that are non-responsive due to anesthesia or trauma retain their capacity for conscious experiences. One candidate marker, suggested to reflect conscious experience (i.e., content consciousness), is the perturbational complexity index (PCI), which reliably discriminates between lower "levels of consciousness", including sleep, anesthesia, and in patients with consciousness disorders (Casali et al., 2013; Sarasso et al., 2015). To our knowledge, the PCI and other candidate markers do not take changes in non-conscious neural processing into account. Again, the lack of specificity, i.e., the inability to isolate neural processing specifically related to (the content of) consciousness and to exclude effects emanating from changes in non-conscious neural processing, is problematic for existing markers of consciousness. The same goes for research on the neural correlates of consciousness where manipulations of arousal are used (Koch et al., 2016). That is, given our current findings, changes in markers/indices or correlates may have been driven by alterations in non-conscious rather than conscious neural processes. While it is possible for non-conscious processes to occur without cooccuring conscious processing, it is impossible for conscious processes to occur without non-conscious processes. Accordingly, non-conscious processes will always need to be accounted for when studying consciousness (Eriksson et al., 2020). For practical purposes, e.g., when trying to determine if a patient is capable of conscious experiences or not, a correlation between arousal and the capacity for conscious experiences may suffice, but should be verified.

Previous neuroimaging research on the effects of sedation has demonstrated a sparing of neural activity in sensory regions combined with a reduction in higher-order regions, including frontal and parietal cortex (Demertzi et al., 2019; Hudetz and Mashour, 2016). Such previous findings are consistent with our current finding of altered BOLD signal in the right IPS with no concomitant signal change in sensory regions during conscious perception. A crucial difference between the current experiment and previous research is that we here directly compare conscious and non-conscious neural processing, while also controlling for non-specific effects from sedation by subtracting BOLD signal during the "absent" condition. While the current study is the first to manipulate both arousal and conscious perception simultaneously, we have only used two levels of sedation. It therefore remains unknown whether the relation is linear or non-linear, and may differ for conscious and non-conscious neural processing. Further characterization of the effects of arousal on conscious and non-conscious neural processing is an important task for future research.

The two main approaches to study the neural correlates of consciousness, by manipulating "level"/"state" or by manipulating "content", have different pros and cons (Dehaene and Changeux, 2011; Koch et al., 2016). The information gained by manipulating content is highly spe- 
cific and excludes many important confounds, but will be limited to the specific content used and cannot provide information on consciousness "in general" (if there is such a thing). Manipulating level/state can provide information in a much broader sense, but lacks control for effects unrelated to consciousness. Recent efforts to improve specificity, for example by looking at similarities across sedatives and other altered states of consciousness (e.g., Huang et al., 2018; Pal et al., 2020; Scheinin et al., 2021, 2018) show promise, but has not yet managed to take non-conscious neural processing into account, as far as we know. Here we combine the two approaches, thereby providing new insights into the effects of manipulating arousal (i.e., the "level" of consciousness). However, with this combined approach comes also the drawback of manipulating the content, i.e., the results are informative only with regard to the specific content that is being manipulated. Thus, generalizability of the current findings is limited by the specifics of the experimental protocol. Further investigations of how the current findings generalize to other stimuli, tasks, and manipulations of arousal, are needed.

Moderate sedation reduced the number of trials reported as subjectively seen (i.e., given a PAS rating > 1) both for "conscious" and "nonconscious" presentation conditions. Without additional measures such as eye-movement recordings or pupillometry it is difficult to know why, but we speculate that it may reflect an increased frequency of attentional lapses and/or eye blinks. The possibility of such a non-specific factor affecting subjective ratings, considered together with findings that (presumably non-conscious) pre-stimulus brain activity affects which stimuli are seen/unseen (He, 2018), makes the change in PAS response distribution itself unsuitable for drawing conclusions regarding conscious and non-conscious processing. Relatedly, the lack of a significant difference in PAS responses as a function of sedation for Absent trials suggest that the criterion for reporting awareness did not change with moderate sedation. Also, the relatively high number of Absent trials with a reported conscious experience (PAS > 1) during both low and moderate sedation (ca 13\%, see Table 1) suggests that the participants used a liberal criterion for reporting awareness.

There exists a relatively large number of theories of consciousness, and an exposition on how the current findings relate to each of them is beyond the scope of the current paper. We refer the interested reader to a recent theory review that also considers levels of consciousness (Northoff and Lamme, 2020). Notably, a key implication from our current findings is that the assumption that altered arousal can be straightforwardly used to elucidate (content) consciousness is problematic, as argued above. A large body of empirical work relevant for various theories of consciousness would therefore need reconsideration. We note also that arousal is but one connotation of "levels of consciousness", and that other conceptions may have better relevance for understanding the complex phenomenon of consciousness.

In conclusion, our current results show that Propofol alters conscious and non-conscious stimulus processing differently. Specifically, non-conscious neural processes were altered in sensory regions while conscious neural processing was altered in a higher-order region. Interestingly, both effects were related to a lateralized response to stimuli appearing in different spatial locations (left/right). By considering sedative effects on non-conscious as well as on conscious neural processing in future work, markers of (content) consciousness can be improved and may lead to a better understanding of situations where content and level dissociate, for example when sedated patients retain the capacity for conscious experiences. We suggest that such future work would benefit from avoiding the term "level of consciousness" when referring to arousal, to reduce confusion.

\section{Data availability statement}

Access to data by qualified investigators is subject to ethical review and must comply with the European Union General Data Protection Regulations/all relevant guidelines. The completion of a data transfer agreement signed by an institutional official will be required.

\section{Declaration of Competing Interest}

The authors declare that they have no competing interests.

\section{Credit authorship contribution statement}

A. Fontan: Methodology, Software, Formal analysis, Investigation, Data curation, Writing - original draft, Visualization. L. Lindgren: Methodology, Investigation, Resources, Writing - review \& editing, Funding acquisition. T. Pedale: Methodology, Software, Writing - review \& editing. C. Brorsson: Investigation, Supervision, Writing - review \& editing. F. Bergström: Methodology, Writing - review \& editing. J. Eriksson: Conceptualization, Methodology, Formal analysis, Resources, Writing - review \& editing, Visualization, Supervision, Project administration, Funding acquisition.

\section{Acknowledgments}

We thank Göran Westling for engineering support, Anders Lundquist for statistical advice, Anders Wåhlin for support with the analysis of ASL data, and Stefan Lehtipalo and Johan Söderberg for support during pilot studies. We also thank Georg Northoff, Christof Koch, and Lars Nyberg for comments on previous versions of the manuscript, and all staff at UFBI for their contribution. This study was supported by Riksbankens Jubileumsfond (Swedish foundation for humanities and social sciences; Grant: P17-0772:1). FB was supported by Fundação para a Ciência e Tecnologia (CEECIND/03661/2017).

\section{References}

Andersson, J.L.R., Hutton, C., Ashburner, J., Turner, R., Friston, K., 2001. Modeling geometric deformations in EPI time series. Neuroimage 13, 903-919.

Aru, J., Suzuki, M., Larkum, M.E., 2020. Cellular mechanisms of conscious processing. Trends Cogn. Sci. 24, 814-825.

Aru, J., Suzuki, M., Rutiku, R., Larkum, M.E., Bachmann, T., 2019. Coupling the state and contents of consciousness. Front. Syst. Neurosci. 13, 1-9.

Ashburner, J., 2007. A fast diffeomorphic image registration algorithm. Neuroimage 38, 95-113.

Axelrod, V., Bar, M., Rees, G., 2014. Exploring the unconscious using faces. Trends Cogn. Sci. 19, 35-45

Bachmann, T., 2012. How to begin to overcome the ambiguity present in differentiation between contents and levels of consciousness? Front. Psychol. 82, 1-6 3.

Bachmann, T., Hudetz, A.G., 2014. It is time to combine the two main traditions in the research on the neural correlates of consciousness: C=LxD. Front. Psychol. 5, 1-13.

Bayne, T., Hohwy, J., Owen, A.M., 2016. Are there levels of consciousness? Trends Cogn. Sci. 20, 405-413.

Bayne, T., Seth, A.K., Massimini, M., 2020. Are there islands of awareness? Trends Neurosci. 43, 6-16.

Begg, C.B., Gray, R., 1984. Calculation of Polychotomous logistic regression parameters using individualized regressions. Biometrika 71, 11-18.

Behzadi, Y., Restom, K., Liau, J., Liu, T.T., 2007. A component based noise correction method (CompCor) for BOLD and perfusion based fMRI. Neuroimage 37, 90-101.

Casali, A.G., Gosseries, O., Rosanova, M., Boly, M., Sarasso, S., Casali, K.R., Casarotto, S., Bruno, M.A., Laureys, S., Tononi, G., Massimini, M., 2013. A theoretically based index of consciousness independent of sensory processing and behavior. Sci. Transl. Med. 5, 198 ra105.

Corbetta, M., Shulman, G.L., 2011. Spatial neglect and attention networks. Annu. Rev. Neurosci. 34, 569-599.

De Schotten, M.T., Dell'Acqua, F., Forkel, S.J., Simmons, A., Vergani, F., Murphy, D.G.M. Catani, M., 2011. A lateralized brain network for visuospatial attention. Nat. Neurosci. $14,1245-1246$.

Dehaene, S., Changeux, .J..-P., 2011. Experimental and theoretical approaches to conscious processing. Neuron $70,200-227$.

Demertzi, A., Tagliazucchi, E., Dehaene, S., Deco, G., Barttfeld, P., Raimondo, F., Martial, C., Fernandez-Espejo, D., Rohaut, B., Voss, H.U., Schiff, N.D., Owen, A.M., Laureys, S., Naccache, L., Sitt, J.D., 2019. Human consciousness is supported by dynamic complex patterns of brain signal coordination. Sci. Adv. 5, 7603.

Eriksson, J., Fontan, A., Pedale, T., 2020. Make the unconscious explicit to boost the science of consciousness. Front. Psychol. 11, $260,1-4$.

Errando, C.L., Sigl, J.C., Robles, M., Calabuig, E., García, J., Arocas, F., Higueras, R., Del Rosario, E., López, D., Peiró, C.M., Soriano, J.L., Chaves, S., Gil, F., García-Aguado, R., 2008. Awareness with recall during general anaesthesia: a prospective observational evaluation of 4001 patients. Br. J. Anaesth. 101, 178-185.

Fazekas, P., Overgaard, M., 2016. Multidimensional models of degrees and levels of consciousness. Trends Cogn. Sci. 20, 715-716.

Fernández-Espejo, D., Owen, A.M., 2013. Detecting awareness after severe brain injury. Nat. Rev. Neurosci. 14, 801-809. 
Friston, K.J., Holmes, A.P., Poline, J.B., Grasby, P.J., Williams, S.C.R., Frackowiak, R.S.J., Turner, R., 1995. Analysis of fMRI time-series revisited. Neuroimage 2, 45-53.

He, B.J., 2018. Robust, transient neural dynamics during conscious perception. Trends Cogn. Sci. 22, 563-565.

Heinke, W., Schwarzbauer, C., 2001. Subanesthetic isoflurane affects task-induced brain: a functional magnetic resonance imaging study. Anesthesiology 94, 973-981.

Hohwy, J., 2009. The neural correlates of consciousness: new experimental approaches needed? Conscious. Cogn. 18, 428-438.

Holmes, A.P., Friston, K.J., 1998. Generalisability, random effects \& population inference. Neuroimage 7, S754.

Huang, Z., Liu, X., Mashour, G.A., Hudetz, A.G., 2018. Timescales of intrinsic BOLD signal dynamics and functional connectivity in pharmacologic and neuropathologic states of unconsciousness. J. Neurosci. 38, 2304-2317.

Hudetz, A.G., Mashour, G.A., 2016. Disconnecting consciousness: is there a common anesthetic end point? Anesth. Analg. 123, 1228-1240.

Jewell, G., Mccourt, M.E., 2000. Pseudoneglect: a review and meta-analysis of performance factors in line bisection tasks. Neuropsychologia 38, 93-110.

Johnston, A.J., Steiner, L.A., Chatfield, D.A., Coleman, M.R., Coles, J.P., Al-Rawi, P.G., Menon, D.K., Gupta, A.K., 2003. Effects of propofol on cerebral oxygenation and metabolism after head injury. Br. J. Anaesth. 91, 781-786.

Koch, C., Massimini, M., Boly, M., Tononi, G., 2016. Neural correlates of consciousness: progress and problems. Nat. Rev. Neurosci. 17, 307-321.

Kouider, S., de Gardelle, V., Sackur, J., Dupoux, E., 2010. How rich is consciousness? The partial awareness hypothesis. Trends Cogn. Sci. 14, 301-307.

Kouider, S., Dehaene, S., 2007. Levels of processing during non-conscious perception: a critical review of visual masking. Philos. Trans. R. Soc. Lond. B. Biol. Sci. 362, 857-875.

Laureys, S., 2005. The neural correlate of (un)awareness: lessons from the vegetative state. Trends Cogn. Sci. 9, 556-559.

Luria, R., Balaban, H., Awh, E., Vogel, E.K., 2016. The contralateral delay activity as a neural measure of visual working memory. Neurosci. Biobehav. Rev. 62, 100-108.

Macdonald, A.A., Naci, L., Macdonald, P.A., Owen, A.M., 2015. Anesthesia and neuroimaging: investigating the neural correlates of unconsciousness. Trends Cogn. Sci. 19, 100-107.

Mashour, G.A., Hudetz, A.G., 2017. Bottom-up and top-down mechanisms of general anesthetics modulate different dimensions of consciousness. Front. Neural Circuits 11, 1-6.

Miller, E.K., Buschman, T.J., 2013. Cortical circuits for the control of attention. Curr. Opin. Neurobiol. 23, 216-222.

Nagel, T., 1974. What is it like to be a bat? Philos. Rev. 83, 435.

Northoff, G., Huang, Z., 2017. How do the brain's time and space mediate consciousness and its different dimensions? Temporo-spatial theory of consciousness (TTC). Neurosci. Biobehav. Rev. 80, 630-645.

Northoff, G., Lamme, V., 2020. Neural signs and mechanisms of consciousness: is there a potential convergence of theories of consciousness in sight? Neurosci. Biobehav. Rev. $118,568-587$.

O'Shea, S.M., Wong, L.C., Harrison, N.L., 2000. Propofol increases agonist efficacy at the GABA a receptor. Brain Res. 852, 344-348.

Overgaard, M., Rote, J., Mouridsen, K., Ramsøy, T.Z., 2006. Is conscious perception gradual or dichotomous? A comparison of report methodologies during a visual task. Conscious. Cogn. 15, 700-708.

Pal, D., Li, D., Dean, J.G., Brito, M.A., Liu, T., Fryzel, A.M., Hudetz, A.G., Mashour, G.A., 2020. Level of consciousness is dissociable from electroencephalographic measures of cortical connectivity, slow oscillations, and complexity. J. Neurosci. 40, 605-618.

Petersen, S.E., Posner, M.I., 2012. The attention system of the human brain: 20 years after. Annu. Rev. Neurosci. 35, 73-89.
Phillips, W.A., Bachmann, T., Storm, J.F., 2018. Apical function in neocortical pyramidal cells : a common pathway by which general anesthetics can affect mental state. Front. Neural Circuits 12, 1-15.

Qiu, M., Scheinost, D., Ramani, R., Constable, R.T., 2017. Multi-modal analysis of functional connectivity and cerebral blood flow reveals shared and unique effects of propofol in large-scale brain networks. Neuroimage 148, 130-140.

Ramsøy, T., Overgaard, M., 2004. Introspection and subliminal perception. Phenomenol. Cogn. Sci. 3, 1-23.

Ratcliff, R., 1993. Methods for dealing with reaction time outliers. Psychol. Bull. 114, 510-532.

Sarasso, S., Boly, M., Napolitani, M., Tononi, G., Laureys, S., Massimini, M., 2015. Consciousness and complexity during unresponsiveness induced by propofol, xenon, and ketamine. Curr. Biol. 25, 3099-3105.

Scheinin, A., Kallionpää, R., Li, D., Kallioinen, M., Kaisti, K., Långsjö, J., Maksimow, A., Vahlberg, T., Valli, K., Mashour, G.A., Revonsuo, A., Scheinin, H., 2018. Differentiating drug-related and state-related effects of dexmedetomidine and propofol on the electroencephalogram. Anesthesiology 129, 22-36.

Scheinin, A., Kantonen, O., Alkire, M., Långsjö, J., Kallionpää, R.E., Kaisti, K., Radek, L., Johansson, J., Sandman, N., Nyman, M., Scheinin, M., 2021. Foundations of human consciousness: imaging the twilight zone. J. Neurosci. 41, 1769-1778.

Searle, J.R., 2000. Consciousness. Annu. Rev. Neurosci. 23, 557-578.

Shulman, G.L., Pope, D.L.W., Astafiev, S.V., Mcavoy, M.P., Snyder, A.Z., Corbetta, M., 2010. Right hemisphere dominance during spatial selective attention and target detection occurs outside the dorsal frontoparietal network. J. Neurosci. 30, 3640-3651.

Siman-Tov, T., Mendelsohn, A., Schonberg, T., Avidan, G., Podlipsky, I., Pessoa, L., Gadoth, N., Ungerleider, L.G., Hendler, T., 2007. Bihemispheric leftward bias in a visuospatial attention- related network. J. Neurosci. 27, 11271-11278.

Sterzer, P., Stein, T., Ludwig, K., Rothkirch, M., Hesselmann, G., 2014. Neural processing of visual information under interocular suppression: a critical review. Front. Psychol. $5,453$.

Suzuki, M., Larkum, M.E., 2020. General anesthesia decouples cortical pyramidal neurons. Cell 180, 666-676.

Thut, G., Nietzel, A., Brandt, S.A., Pascual-Leone, A., 2006. $\alpha$-band electroencephalographic activity over occipital cortex indexes visuospatial attention bias and predicts visual target detection. J. Neurosci. 26, 9494-9502.

Tononi, G., Boly, M., Massimini, M., Koch, C., 2016. Integrated information theory: from consciousness to its physical substrate. Nat. Rev. Neurosci. 17, 450-461.

Trapani, G.M., Altomare, C., Sanna, E., Biggio, G., Liso, G., 2000. Propofol in anesthesia mechanism of action, structure-activity relationships, and drug delivery. Curr. Med. Chem. 7, 249-271.

Tsuchiya, N., Koch, C., 2005. Continuous flash suppression reduces negative afterimages. Nat. Neurosci. 8, 1096-1101.

Veselis, R.A., Feshchenko, V.A., Reinsel, R.A., Beattie, B., Akhurst, T.J., 2005. Propofol and thiopental do not interfere with regional cerebral blood flow response at sedative concentrations. Anesthesiology 102, 26-34.

Vuilleumier, P., 2013. Mapping the functional neuroanatomy of spatial neglect and human parietal lobe functions: progress and challenges. Ann. N. Y. Acad. Sci. 1296, 50-74.

Wang, C., Ding, M., Kluger, B.M., 2014. Change in intraindividual variability over time as a key metric for defining performance-based cognitive fatigability. Brain Cogn. 85, 251-258.

Wang, L., Mruczek, R.E.B., Arcaro, M.J., Kastner, S., 2015. Probabilistic maps of visual topography in human cortex. Cereb. Cortex 25, 3911-3931.

Wislowska, M., Giudice, R., Lechinger, J., Wielek, T., Heib, P.J., Pitiot, A., Pichler, G. Michitsch, G., Donis, J., 2017. Night and day variations of sleep in patients with disorders of consciousness. Sci. Rep. 7, 1-11. 